\title{
The nilpotent cone in the Mukai system of rank two and genus two
}

\author{
Isabell Hellmann ${ }^{1}$
}

Received: 18 November 2019 / Revised: 25 February 2021 / Accepted: 2 March 2021 /

Published online: 30 March 2021

(c) The Author(s) 2021

\section{Abstract}

We study the nilpotent cone in the Mukai system of rank two and genus two. We compute the degrees and multiplicities of its irreducible components and describe their cohomology classes.

\section{Introduction}

Let $(S, H)$ be a polarized $\mathrm{K} 3$ surface of genus $g$ and fix two coprime integers $n \geq 1$ and $s$. The moduli space $M=M_{H}(v)$ of $H$-Gieseker stable coherent sheaves with Mukai vector $v=(0, n H, s)$ is a smooth Hyperkähler variety of dimension $2\left(n^{2}(g-1)+1\right)$. A point in $M$ corresponds to a stable sheaf $\mathcal{E}$ on $S$ such that $\mathcal{E}$ is pure of dimension one with support in the linear system $|n H|$. Taking the (Fitting) support defines a Lagrangian fibration

$$
f: M \longrightarrow|n H| \cong \mathbb{P}^{n^{2}(g-1)+1},[\mathcal{E}] \mapsto \operatorname{Supp}(\mathcal{E})
$$

known as the Mukai system [5,22]. Over a general point in $|n H|$ which corresponds to a smooth curve $D \subset S$ the fibers of $f$ are abelian varieties isomorphic to $\operatorname{Pic}^{\delta}(D)$, where $\delta=s-n^{2}(1-g)$. So, $M$ can also be viewed as a relative compactified Jacobian associated to the universal curve $\mathcal{C} \rightarrow|n H|$.

The Mukai system is of special interest because of its relation to the classical and widely studied Hitchin system, see [14] for a survey. Let $C$ be a smooth curve of

Communicated by Vasudevan Srinivas.

The author is supported by the SFB/TR 45 'Periods, Moduli Spaces and Arithmetic of Algebraic varieties' of the DFG (German Research Foundation, German Government's Excellence Initiative) and the Bonn International Graduate School.

$凶 \quad$ Isabell Hellmann

igb@math.uni-bonn.de

1 Mathematisches Institut, Universität Bonn, Endenicher Allee 60, 53115 Bonn, Germany 
genus $g$. A Higgs bundle on $C$ is a pair $(\mathcal{E}, \phi)$ consisting of a vector bundle $\mathcal{E}$ on $C$ and a morphism $\phi: \mathcal{E} \rightarrow \mathcal{E} \otimes \omega_{C}$, called Higgs field. The moduli space $M_{\text {Higgs }}(n, d)$ of stable Higgs bundles of rank $n$ and degree $d$ is a smooth and quasi-projective symplectic variety. Sending $(\mathcal{E}, \phi)$ to the coefficients of its characteristic polynomial $\chi(\phi)$ defines a proper Lagrangian fibration

$$
\chi: M_{\text {Higgs }}(n, d) \longrightarrow \bigoplus_{i=1}^{n} H^{0}\left(C, \omega_{C}^{i}\right)
$$

It is equivariant with respect to the $\mathbb{C}^{*}$-action that is given by scaling the Higgs field on $M_{\text {Higgs }}(n, d)$ and by multiplication with $t^{i}$ in the corresponding summand on the base. As a corollary the topology of $M_{\text {Higgs }}(n, d)$ is controlled by the fiber over the origin. This fiber

$$
N:=\chi^{-1}(0)=\left\{(\mathcal{E}, \phi) \in M_{\text {Higgs }}(n, d) \mid \phi \text { is nilpotent }\right\}
$$

is called the nilpotent cone. In the late ' 80 s Beauville, Narasimhan, and Ramanan discovered a beautiful interpretation of the space of Higgs bundles [4]. They showed that a Higgs bundle $(\mathcal{E}, \phi)$ with characteristic polynomial $s$ corresponds to a pure sheaf of rank one on a so called spectral curve $C_{S} \subset T^{*} C$ inside the cotangent bundle of $C$. The curve $C_{s}$ is defined in terms of $s=\chi(\phi)$ and is linearly equivalent to $n C$, the $n$-th order thickening of the zero section $C \subset T^{*} C$. This idea was taken up by Donagi, Ein, and Lazarsfeld in [9]: The space $M_{\text {Higgs }}(n, d)$ appears as a moduli space of stable sheaves on $T^{*} C$ that are supported on curves in the linear system $|n C|$. Consequently, $M_{\text {Higgs }}(n, d)$ has a natural compactification $\bar{M}_{\text {Higgs }}(n, d)$ given by a moduli space of sheaves on the projective surface $S_{0}=\mathbb{P}\left(\omega_{C} \oplus \mathcal{O}_{C}\right)$ with respect to the polarization $H_{0}=\mathcal{O}_{S_{0}}(C)$. The Hitchin map extends to $\bar{M}_{\text {Higgs }}(n, d) \rightarrow\left|n H_{0}\right| \cong \mathbb{P}\left(\oplus_{i=0}^{n} H^{0}\left(\omega_{C}^{i}\right)\right)$ and is nothing but the support map; the nilpotent cone is the fiber over the point $n C \in\left|n H_{0}\right|$. However, $\bar{M}_{\text {Higgs }}(n, d)$ cannot admit a symplectic structure as it is covered by rational curves. At this point the Mukai system enters the picture. If $S$ is a K3 surface that contains the curve $C$ as a hyperplane section, one can degenerate $(S, H)$ to $\left(S_{0}, H_{0}\right)$ and consequently the Mukai system $M_{H}(v) \rightarrow|n H|$ with $v=(0, n H, d+n(1-g))$ degenerates to the compactified Hitchin system $[9, \S 1]$. From our perspective, this is a powerful approach to studying the Hitchin system. For instance, in a recent paper [7], de Cataldo, Maulik and Shen prove the $\mathrm{P}=\mathrm{W}$ conjecture for $g=2$ by means of the corresponding specialization map on cohomology.

In this note, we study the geometry of the nilpotent cone in the Mukai system, which is defined in parallel to the Hitchin system

$$
N_{C}:=f^{-1}(n C),
$$

for some curve $C \in|H|$. Alternatively, one could say that we study the most singular fiber type, see (3.1). We will fix the invariants $n=2$ and $g=2$ and the Mukai vector $v=(0,2 H,-1)$. In this case and if $C$ is irreducible, the nilpotent cone has two 
irreducible components

$$
\left(N_{C}\right)_{\text {red }}=N_{0} \cup N_{1},
$$

where the first component is isomorphic to the moduli space $M_{C}(2,1)$ of stable vector bundles of rank two and degree one on $C$ and the second component is the closure of $N_{C} \backslash N_{0}$. Both components are Lagrangian subvarieties of $M=M_{H}(v)$. If $C$ is smooth, then $N_{0}$ is smooth and the singularities of $N_{1}$ are contained in $N_{0} \cap N_{1}$ (each understood with their reduced structure). However, both components occur with multiplicities.

Our first result is the computation of the multiplicities of the components as well as their degrees. Here, the degree is meant with respect to a naturally defined distinguished ample class $u_{1} \in H^{2}(M, \mathbb{Z})$, see Definition 4.7.

Theorem 1.1 Let $C \in|H|$ be an irreducible curve. The degrees of the two components of the nilpotent cone $N_{C}$ are given by

$$
\operatorname{deg}_{u_{1}} N_{0}=5 \cdot 2^{9} \text { and } \operatorname{deg}_{u_{1}} N_{1}=5^{2} \cdot 2^{11}
$$

and their multiplicities are

$$
\operatorname{mult}_{N_{C}} N_{0}=2^{3} \text { and } \text { mult }_{N_{C}} N_{1}=2 \text {. }
$$

Moreover, any fiber $F$ of the Mukai system has degree $5 \cdot 3 \cdot 2^{13}$.

As the smooth locus of every component with its reduced structure deforms from the Mukai to the Hitchin system, the multiplicities and degrees must coincide. Here, indeed, the same multiplicities can be found in [23, Propositions 34 and 35] and [15, Proposition 6], whereas, up to our knowledge, the degrees have not been determined in the literature. In our case, the degrees determine the multiplicities.

Our second result is a description of the classes $\left[N_{0}\right]$ and $\left[N_{1}\right] \in H^{10}(M, \mathbb{Z})$. The projective moduli spaces of stable sheaves on K3 surfaces are known to be deformation equivalent to Hilbert schemes of points. In our case, $M$ is actually birational to $S^{[5]}[6$, Lemma 3.2.7]. In particular, there is an isomorphism $H^{*}(M, \mathbb{Z}) \cong H^{*}\left(S^{[5]}, \mathbb{Z}\right)$. The cohomology ring of $S^{[5]}$ is well understood, e.g. [19, §4] and the references therein. Recall that for any Hyperkähler variety $X$ of dimension $2 n$ there is an embedding $S^{i} H^{2}(X, \mathbb{Q}) \hookrightarrow H^{2 i}(X, \mathbb{Q})$ for all $i \leq n[24$, Theorem 1.7].

Theorem 1.2 The classes $\left[N_{0}\right]$ and $\left[N_{1}\right] \in H^{10}(M, \mathbb{Q})$ are linearly independent and span a totally isotropic subspace of $H^{10}(M, \mathbb{Q})$ with respect to the intersection pairing. They are given by

$$
\left[N_{0}\right]=\frac{1}{48}[F]+\beta \text { and }\left[N_{1}\right]=\frac{5}{12}[F]-4 \beta,
$$

where $[F]$ is the class of a general fiber of the Mukai system and $0 \neq \beta \in$ $\left(S^{5} H^{2}(M, \mathbb{Q})\right)^{\perp}$ satisfies $\beta^{2}=0$. As $\operatorname{deg}_{u_{1}} \beta=0$, the class $\beta$ is not effective. 


\section{Outline}

In Sect. 2 we introduce the Mukai system. In Sect. 3 we reduce the study of $N_{C}$ to the case of a smooth curve $C$. We describe the irreducible components of the nilpotent cone following $[9, \S 3]$, where it is shown that any point $[\mathcal{E}] \in N_{C} \backslash N_{0}$ fits into an extension of the form

$$
0 \rightarrow \mathcal{L}(x) \otimes \omega_{C}^{-1} \longrightarrow \mathcal{E} \longrightarrow \mathcal{L} \rightarrow 0
$$

where $\mathcal{L} \in \operatorname{Pic}^{1}(C)$ is a line bundle and $x \in C$ a point. We specify a space $W \rightarrow \operatorname{Pic}^{1}(C) \times C$ parameterizing such extensions, and a compactification $\bar{W}$ of $W$ that comes with a birational map $v: \bar{W} \rightarrow N_{1}$. In the Hitchin case, this idea originates from [23].

In Sect. 4 we prove Theorem 1.1. The proof relies on the functorial properties of the defintion of $u_{1}$ via the determinant line bundle construction, see Sect. 4.1. It allows us to relate $\left.u_{1}\right|_{F}$ and $\left.u_{1}\right|_{N_{0}}$ with the (generalized) theta divisor on $F=f^{-1}(D) \cong \operatorname{Pic}^{3}(D)$ for $D \in|n C|$ smooth and $M_{C}(2,1)$, respectively, see Propositions 4.8 and 4.10. For $\left[N_{1}\right]$ the degree computation is achieved by determining $v^{*} u_{1} \in H^{2}(\bar{W}, \mathbb{Z})$. Finally, the multiplicities are infered from knowing the degrees. The last Sect. 5 is devoted to the proof of Theorem 1.2. It uses our previous results.

\section{Notation}

All schemes are of finite type over $k=\mathbb{C}$. In the entire paper, $S$ is a K3 surface polarized by a primitive, ample class $H \in \mathrm{NS}(S)$ with $H^{2}=2 g-2$.

\section{The Mukai system}

In this section, we give a brief recollection on moduli spaces of sheaves on K3 surfaces and define the Mukai system. First recall that the Mukai vector induces an isomorphism

$$
v: \mathrm{K}(S)_{\text {num }} \stackrel{\sim}{\rightarrow} H_{\mathrm{alg}}^{*}(S, \mathbb{Z})=H^{0}(S, \mathbb{Z}) \oplus \mathrm{NS}(S) \oplus H^{4}(S, \mathbb{Z}) .
$$

It is given by

$$
v(\mathcal{E}):=\operatorname{ch}(\mathcal{E}) \sqrt{\operatorname{td}(S)}=\left(\operatorname{rk}(\mathcal{E}), c_{1}(\mathcal{E}), \chi(\mathcal{E})-\operatorname{rk}(\mathcal{E})\right)
$$

We write $M_{H}(v)$ for the moduli space of pure, $H$-Gieseker stable sheaves on $S$ with Mukai vector $v$. If $v$ is primitive and positive and $H$ is $v$-generic then $M_{H}(v)$ is an irreducible holomorphic symplectic manifold of dimension $\langle v, v\rangle+2$, which is deformation equivalent to the Hilbert scheme of $\frac{1}{2}\langle v, v\rangle+1$ points on $S[18$, Theorem 10.3.1]. Here, $\langle$,$\rangle is the Mukai pairing given by$

$$
\left\langle(r, c, s),\left(r^{\prime}, c^{\prime}, s^{\prime}\right)\right\rangle=c c^{\prime}-r s^{\prime}-r^{\prime} s .
$$


Consider the Mukai vector

$$
v:=(0, n H, s) \in H_{\mathrm{alg}}^{*}(S, \mathbb{Z}),
$$

and assume that $v$ is primitive. A pure sheaf $\mathcal{F}$ of Mukai vector $v$ has one-dimensional support, first Chern class $n H$ and Euler characteristic $s$. In particular, $\mathcal{F}$ admits a length one resolution $0 \rightarrow \mathcal{V} \stackrel{f}{\longrightarrow} \tilde{\mathcal{V}} \longrightarrow \mathcal{F}$ by two vector bundles of the same rank $r[16, \S 1.1]$. We define the (Fitting) support of $\mathcal{F}$ to be

$$
\operatorname{Supp}(\mathcal{F}):=V(\operatorname{det} f) \subset S
$$

the vanishing scheme of the induced morphism det $f=\wedge^{r} f: \wedge^{r} \mathcal{V} \rightarrow \wedge^{r} \tilde{\mathcal{V}}$, for any resolution $0 \rightarrow \mathcal{V} \stackrel{f}{\rightarrow} \tilde{\mathcal{V}}$ of $\mathcal{F}$ as above. This definition is well-defined, i.e. independent of the chosen resolution [13, Definition 20.4].

Example 2.1 Let $i: C \hookrightarrow S$ be an integral curve and $\mathcal{E}$ a vector bundle of rank $n$ on $C$. Then

$$
\operatorname{Supp}\left(i_{*} \mathcal{E}\right)=n C
$$

is the $n$-th order thickening of $C$ in $S$.

By definition, $\operatorname{Supp}(\mathcal{F})$ is linearly equivalent to $c_{1}(\mathcal{F})$ and $\operatorname{Supp}(\mathcal{F})$ contains the usual support defined by the annihilator of $\mathcal{F}$. Moreover, the reduced locus $\operatorname{Supp}(\mathcal{F})_{\text {red }}$ is the set-theoretic support of $\mathcal{F}$. The advantage of the above definition is, that it behaves well in families and thus induces a morphism [20, §2.2]

$$
f: M_{H}(v) \longrightarrow|n H| \cong \mathbb{P}^{\tilde{g}}, \quad[\mathcal{E}] \mapsto \operatorname{Supp}(\mathcal{E})
$$

Here, $\tilde{g}=n^{2}(g-1)+1$. Moreover, $M_{H}(v)$ is irreducible holomorphic symplectic of dimension $n^{2} H^{2}+2=2 \tilde{g}$ and hence, by Matsushita's result [21, Corollary 1] this morphism is a Lagrangian fibration (for an explicit proof see [9, Lemma 1.3]), called the Mukai system (of rank $n$ and genus $g$ ).

\section{The nilpotent cone for $n=2$ and $g=2$}

We now specialize to the case that $n=2$ and $s=3-2 g$ with $g=2$, i.e. we fix the Mukai vector

$$
v=(0,2 H,-1) \text {. }
$$

In particular, a stable vector bundle of rank two and degree one on a smooth curve $C \in|H|$ defines a point in $M:=M_{H}(v)$. We have $\operatorname{dim} M=8 g-6=10$ and $M$ is birational to the Hilbert scheme $S^{[5]}$ of five points on $S$. 
Taking (Fitting) supports defines a Lagrangian fibration

$$
f: M \longrightarrow|2 H| \cong \mathbb{P}^{5}
$$

We have a natural morphism $|H| \times|H| \rightarrow|2 H|$. We define $\Sigma \subset|2 H|$ as its image and $\Delta \subset \Sigma$ as the image of the diagonal. Then $\Sigma \cong \operatorname{Sym}^{2}|H|$ and $\Delta \cong|H|$. If every curve in $|H|$ is irreducible (e.g. if $\operatorname{Pic}(S)=\mathbb{Z} \cdot H$ ) then $\Delta$ and $\Sigma$ are exactly the loci of non-reduced and non-integral curves, respectively. And in this case we can distinguish three cases following [6, Proposition 3.7.1]:

$$
f^{-1}(x) \begin{cases}\text { is reduced and irreducible } & \text { if } x \in|2 H| \backslash \Sigma \\ \text { is reduced and has two irreducible components } & \text { if } x \in \Sigma \backslash \Delta \\ \text { has two irreducible components with multiplicities } & \text { if } x \in \Delta .\end{cases}
$$

In the general case, the list is still valid for for the geometric generic point in the respective subvariety. However, over points that correspond to curves with more irreducible components, one also finds more irreducible components in the fiber [6, Proof of Lemma 3.3.2].

We will study fibers of the third type, namely

$$
N_{C}:=f^{-1}(2 C) \text {, }
$$

where $C \in|H|$ is irreducible. In analogy with the Hitchin system, we call $N_{C}$ nilpotent cone.

For the rest of the paper, we fix a smooth curve $C \in|H|$ and write $N$ instead of $N_{C}$. We will now identify the irreducible components of $N$ following the ideas of [9].

\subsection{Pointwise description of the nilpotent cone $N=N_{C}$}

Let $[\mathcal{E}] \in N$ and consider its restriction $\left.\mathcal{E}\right|_{C}$ to $C$. There are two cases, either $\left.\mathcal{E}\right|_{C}$ is a stable rank two vector bundle on $C$ or $\left.\mathcal{E}\right|_{C}$ has rank one. By dimension reasons, the sheaves of the first kind contribute an irreducible component $N_{0}$ of $N$ isomorphic to the moduli space $\mathrm{M}_{C}(2,1)$ of stable rank two and degree one vector bundles on $C$. In the second case, $\left.\mathcal{E}\right|_{C} \cong \mathcal{L} \oplus \mathcal{O}_{D}$, where the first factor $\mathcal{L}:=\left.\mathcal{E}\right|_{C} /$ torsion is a line bundle on $C$ and $D \subset C$ is an effective divisor. We set

$$
E_{1}:=N \backslash N_{0}
$$

with reduced structure.

Lemma 3.1 Let $[\mathcal{E}] \in E_{1}$ and write $\left.\mathcal{E}\right|_{C}=\mathcal{L} \oplus \mathcal{O}_{D}$. There is a short exact sequence of $\mathcal{O}_{S}$-modules

$$
0 \rightarrow i_{*}\left(\mathcal{L}(D) \otimes \omega_{C}^{-1}\right) \longrightarrow \mathcal{E} \longrightarrow i_{*} \mathcal{L} \rightarrow 0 .
$$

Moreover, $k:=\operatorname{deg} \mathcal{L}=1$ and $d:=\operatorname{deg} D=2 g-2 k-1=1$. 
Proof Noting that $\omega_{C}^{-1}$ is the conormal bundle of $C$ in $S$, it is straightforward to obtain the sequence (3.2). Let us prove the numerical restrictions. From (3.2) we have

$$
1+2(1-g)=\chi(\mathcal{E})=\chi\left(\mathcal{L}(D) \otimes \omega_{C}^{-1}\right)+\chi(\mathcal{L})=2 k+d-(2 g-2)+2(1-g)
$$

Thus $d=2 g-2 k-1$ and we find $k \leq g-1$. On the other hand, $\mathcal{E}$ is stable and therefore the reduced Hilbert polynomials [16, Definition 1.2.3] of $\mathcal{E}$ and $\mathcal{L}$ satisfy $p(\mathcal{E}, t)<p(\mathcal{L}, t)$, which amounts to

$$
\frac{1}{2}(1+2(1-g))<k+1-g
$$

or equivalently $k \geq 1$.

Remark 3.2 For $n=2$ and arbitrary genus $g$, one has $\operatorname{deg} \mathcal{L} \in\{1, \ldots, g-1\}$ and a decomposition into locally closed subsets $N_{\text {red }}=N_{0} \sqcup E_{1} \sqcup \ldots \sqcup E_{g-1}$ corresponding to the degree of $\mathcal{L}$. In fact, $N_{0}$ and the closures of $E_{k}$ are the irreducible components of $N$.

We conclude that every point in $E_{1}$ defines a class in $\operatorname{Ext}_{S}^{1}\left(i_{*} \mathcal{L}, i_{*}\left(\mathcal{L}(x) \otimes \omega_{C}^{-1}\right)\right)$ for some point $x \in C$ and some line bundle $\mathcal{L} \in \operatorname{Pic}^{1}(C)$. Conversely, an extension class in $\operatorname{Ext}_{S}^{1}\left(i_{*} \mathcal{L}, i_{*}\left(\mathcal{L}(x) \otimes \omega_{C}^{-1}\right)\right)$ defines a point in $E_{1}$ if and only if its middle term is stable and has the point $x$ as support of its torsion part when restricted to $C$, i.e. if it is not pushed forward from $C$. It turns out that all such extensions are stable.

Lemma 3.3 Consider a coherent sheaf $\mathcal{E}$ on $S$ that is given as an extension

$$
0 \rightarrow i_{*} \mathcal{L} \rightarrow \mathcal{E} \rightarrow i_{*} \mathcal{L}^{\prime} \rightarrow 0
$$

where $\mathcal{L}^{\prime}$ and $\mathcal{L}$ are line bundles on $C$ of degree $k$ and $1-k$, respectively, with $k \geq 1$. Moreover, assume that $\mathcal{E}$ itself does not admit the structure of an $\mathcal{O}_{C}$-module. Then $\mathcal{E}$ is $H$-Gieseker stable.

Proof We have to prove $p(\mathcal{E}, t)<p(\mathcal{M}, t)$ or, equivalently, $\frac{\chi(\mathcal{E})}{c_{1}(\mathcal{E}) \cdot H}<\frac{\chi(\mathcal{M})}{c_{1}(\mathcal{M}) \cdot H}$ for every surjection $\mathcal{E} \rightarrow \mathcal{M}$. We can assume that $\operatorname{Supp}(\mathcal{M})=C$ and $\mathcal{M}=i_{*} \mathcal{M}^{\prime}$, where $\mathcal{M}^{\prime}$ is a line bundle on $C$. Then because $\left.\mathcal{E}\right|_{C} \cong \mathcal{L}^{\prime} \oplus \mathcal{T}$ for some torsion sheaf $\mathcal{T}$, we find

$$
\mathcal{H o m}_{\mathcal{O}_{S}}\left(\mathcal{E}, i_{*} \mathcal{M}^{\prime}\right) \cong \mathcal{H o m}_{\mathcal{O}_{C}}\left(\left.\mathcal{E}\right|_{C}, \mathcal{M}^{\prime}\right) \cong \mathcal{H o m}_{\mathcal{O}_{C}}\left(\mathcal{L}^{\prime}, \mathcal{M}^{\prime}\right)
$$

and thus $i_{*} \mathcal{L}^{\prime} \stackrel{\sim}{\rightarrow} \mathcal{M}$.

Corollary 3.4 The closed points of $E_{1}$ are in bijection with the following set

$$
\bigsqcup_{\substack{\mathcal{L} \in \operatorname{Pic}^{1}(C) \\ x \in C}} \mathbb{P}\left(\operatorname{Ext}_{S}^{1}\left(i_{*} \mathcal{L}, i_{*}\left(\mathcal{L}(x) \otimes \omega_{C}^{-1}\right)\right)\right) \backslash \mathbb{P}\left(\operatorname{Ext}_{C}^{1}\left(\mathcal{L}, \mathcal{L}(x) \otimes \omega_{C}^{-1}\right)\right),
$$


i.e. with extension classes $[v] \in \mathbb{P}\left(\operatorname{Ext}_{S}^{1}\left(i_{*} \mathcal{L}, i_{*}\left(\mathcal{L}(x) \otimes \omega_{C}^{-1}\right)\right)\right)$ such that $v$ is not pushed forward from $C$. Here, $\mathcal{L}$ varies over all line bundles on $C$ with $\operatorname{deg} \mathcal{L}=1$, and $x$ varies over all points in $C$. The bijection is established by Lemma 3.1.

In Proposition 3.5 below, we will see that there is a short exact sequence

$$
0 \rightarrow \operatorname{Ext}_{C}^{1}\left(\mathcal{L}, \mathcal{L}(x) \otimes \omega_{C}^{-1}\right) \rightarrow \operatorname{Ext}_{S}^{1}\left(i_{*} \mathcal{L}, i_{*}\left(\mathcal{L}(x) \otimes \omega_{C}^{-1}\right)\right) \stackrel{\rho_{\mathcal{L}, x}}{\longrightarrow} H^{0}\left(C, \mathcal{O}_{C}(x)\right) \rightarrow 0,
$$

where $\rho_{\mathcal{L}, x}$ has the following interpretation modulo a scalar factor. If $\mathcal{E}$ is the middle term of a representing sequence of $v \in \operatorname{Ext}_{S}^{1}\left(i_{*} \mathcal{L}, i_{*}\left(\mathcal{L}(x) \otimes \omega_{C}^{-1}\right)\right)$, then

$$
\left.\mathcal{E}\right|_{C} \cong \mathcal{L} \oplus \mathcal{O}_{V\left(\rho_{\mathcal{L}, x}(v)\right)}
$$

Hence, another way to phrase Corollary 3.4 is by fixing for every $x \in C$ a defining section $s_{x} \in H^{0}\left(C, \mathcal{O}_{C}(x)\right)$ as follows. Let $\Delta \hookrightarrow C \times C$ be the diagonal, yielding a section $s_{\Delta} \in H^{0}(C \times C, \mathcal{O}(\Delta))$. For every $x \in C$, we set $s_{x}=\left.s_{\Delta}\right|_{\{x\} \times C}$. Then we can write

$$
\text { points of } E_{1} \stackrel{1: 1}{\longleftrightarrow} \bigsqcup_{\substack{\mathcal{L} \in \operatorname{Pic}^{1}(C) \\ x \in C}}\left\{v \in \operatorname{Ext}_{S}^{1}\left(i_{*} \mathcal{L}, i_{*}\left(\mathcal{L}(x) \otimes \omega_{C}^{-1}\right)\right) \mid \rho_{\mathcal{L}, x}(v)=s_{x}\right\}
$$

\subsection{Extension spaces}

So far, we have given a pointwise description of the nilpotent cone. Next, we will identify its irreducible components and their scheme structures. This subsection is a technical parenthesis in this direction. The reader may like to skip it.

Let $S$ be a smooth projective surface and $i: C \hookrightarrow S$ a smooth curve with normal bundle $\mathcal{N}_{C / S} \cong \mathcal{O}_{C}(C)$. Let $T$ be any scheme and let $\mathcal{F}$ and $\mathcal{F}^{\prime}$ be two vector bundles on $T \times C$ considered as families of vector bundles on $C$. Denote by $\pi: T \times S \rightarrow T$ and $\pi_{C}: T \times C \rightarrow T$ the projections. For a morphism $f: X \rightarrow Y$, we write $\mathcal{E}_{x} t_{f}$ instead of $R f_{*} R \mathcal{H}$ om.

Proposition 3.5 There is a short exact sequence of $\mathcal{O}_{T}$-modules

$$
0 \rightarrow \mathcal{E} x t_{\pi_{C}}^{1}\left(\mathcal{F}^{\prime}, \mathcal{F}\right) \rightarrow \mathcal{E} x t_{\pi}^{1}\left((\mathrm{id} \times i)_{*} \mathcal{F}^{\prime},(\mathrm{id} \times i)_{*} \mathcal{F}\right) \stackrel{\rho}{\rightarrow} \mathcal{E} x t_{\pi_{C}}^{0}\left(\mathcal{F}^{\prime} \otimes \mathcal{O}_{C}(-C), \mathcal{F}\right) \rightarrow 0
$$

as well as for every $t \in T$ a short exact sequence of vector spaces

$$
0 \rightarrow \operatorname{Ext}_{C}^{1}\left(\mathcal{F}_{t}^{\prime}, \mathcal{F}_{t}\right) \stackrel{\xi}{\longrightarrow} \operatorname{Ext}_{S}^{1}\left(i_{*} \mathcal{F}_{t}^{\prime}, i_{*} \mathcal{F}_{t}\right) \stackrel{\rho_{t}}{\longrightarrow} \operatorname{Ext}_{C}^{0}\left(\mathcal{F}_{t}^{\prime} \otimes \mathcal{O}_{C}(-C), \mathcal{F}_{t}\right) \rightarrow 0
$$

Note that the fibers of (3.4) must, in general, not coincide with (3.5), see Lemma 3.6.

Proof Apply $R \pi_{C *} R \mathcal{H} \operatorname{Hom}(, \mathcal{F})$ or $R \operatorname{Hom}\left(, \mathcal{F}_{t}\right)$, to the exact triangle

$$
\mathcal{F}^{\prime} \otimes \mathcal{O}_{C}(-C)[1] \rightarrow L(\mathrm{id} \times i)^{*}(\mathrm{id} \times i)_{*} \mathcal{F}^{\prime} \rightarrow \mathcal{F}^{\prime} \stackrel{[1]}{\longrightarrow}
$$


in $D^{b}(T \times C)\left(\right.$ see $\left[17\right.$, Corollary 11.4]) or its counterpart in $D^{b}(C)$, respectively, and consider the induced cohomology sequence.

We can explicitly describe the morphism $\rho_{t}$ in the sequence (3.5). Represent $v \in$ $\operatorname{Ext}_{S}^{1}\left(i_{*} \mathcal{F}_{t}^{\prime}, i_{*} \mathcal{F}_{t}\right)$ by $0 \rightarrow i_{*} \mathcal{F}_{t} \rightarrow \mathcal{E} \rightarrow i_{*} \mathcal{F}_{t}^{\prime} \rightarrow 0$. Restriction to $C$ yields

$$
\cdots \rightarrow \mathcal{F}_{t}^{\prime} \otimes \mathcal{O}_{C}(-C) \stackrel{\delta(v)}{\longrightarrow} \mathcal{F}_{t} \rightarrow \mathcal{E}_{C} \rightarrow \mathcal{F}_{t}^{\prime} \rightarrow 0
$$

where we inserted

$\mathcal{T}_{\operatorname{or}_{1}}^{\mathcal{O}_{S}}\left(i_{*} \mathcal{F}_{t}^{\prime}, i_{*} \mathcal{O}_{C}\right) \cong \mathcal{F}_{t}^{\prime} \otimes \mathcal{O}_{C} \mathcal{T}_{\text {or }}^{\mathcal{O}_{S}}\left(i_{*} \mathcal{O}_{C}, i_{*} \mathcal{O}_{C}\right)=\mathcal{F}_{t}^{\prime} \otimes \mathcal{O}_{C}(-C)$. This gives a well-defined, linear map

$$
\delta: \operatorname{Ext}_{S}^{1}\left(i_{*} \mathcal{F}_{t}^{\prime}, i_{*} \mathcal{F}_{t}\right) \rightarrow \operatorname{Ext}_{C}^{0}\left(\mathcal{F}_{t}^{\prime} \otimes \mathcal{O}_{C}(-C), \mathcal{F}_{t}\right)
$$

As $\operatorname{im} \xi=\operatorname{ker} \delta$, it follows by dimension reasons, that $\delta$ has to be surjective. So, $\rho_{t}=\delta$ up to post-composition with an isomorphism of $\operatorname{Ext}_{C}^{0}\left(\mathcal{F}_{t}^{\prime} \otimes \mathcal{O}_{C}(-C), \mathcal{F}_{t}\right)$.

Lemma 3.6 For every $t \in T$ there is a commutative diagram of short exact sequences

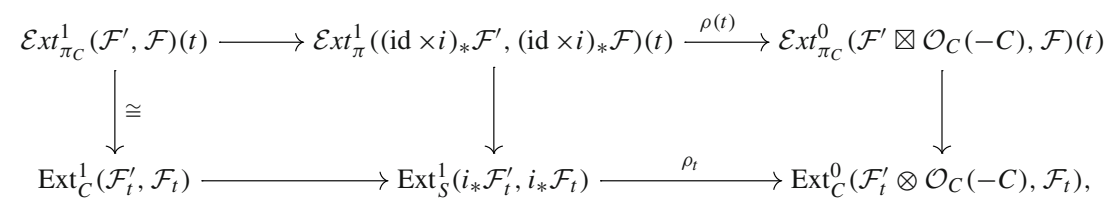

where the first vertical arrow is an isomorphism. If $\operatorname{Ext}_{C}^{0}\left(\mathcal{F}_{t}^{\prime} \otimes \mathcal{O}_{C}(-C), \mathcal{F}_{t}\right)$ has constant dimension for all $t \in T$ all vertical arrows are isomorphisms.

Proof The vertical morphisms are the usual functorial base change morphisms. The lower line is (3.5) and hence also exact on the left. The first vertical arrow is an isomorphism because $\operatorname{Ext}_{C}^{2}\left(\mathcal{F}_{t}^{\prime}, \mathcal{F}_{t}\right)=0$. Consequently, also the upper line is exact on the left.

\subsection{Irreducible components of $\mathbf{N}$}

In this section, we show that $E_{1}$ is irreducible and has the same dimension as $N$. Therefore its closure

$$
N_{1}:=\bar{E}_{1} \subset N_{\text {red }}
$$

with reduced structure is an irreducible component of $N$. For the proof, we need some more notation. Let $\mathcal{P}_{1}$ be a Poincaré line bundle on $\operatorname{Pic}^{1}(C) \times C$ and $\Delta \subset C \times C$ the diagonal. Set $T:=\operatorname{Pic}^{1}(C) \times C$ and on $T$ define the following sheaves

$$
\begin{aligned}
\mathcal{V} & :=R^{1} p_{12 *}\left(p_{23}^{*} \mathcal{O}(\Delta) \otimes p_{3}^{*} \omega_{C}^{-1}\right), \\
\mathcal{W} & :=R^{1} p_{12 *} R \mathcal{H} \operatorname{om}\left((\mathrm{id} \times i)_{*} p_{13}^{*} \mathcal{P}_{1},(\mathrm{id} \times i)_{*}\left(p_{13}^{*} \mathcal{P}_{1} \otimes p_{23}^{*} \mathcal{O}(\Delta) \otimes p_{3}^{*} \omega_{C}^{-1}\right)\right. \text { and } \\
\mathcal{U} & :=p_{12 *} p_{23}^{*} \mathcal{O}(\Delta),
\end{aligned}
$$


where $p_{i j}$ are the appropriate projections from $\operatorname{Pic}^{1}(C) \times C \times C$. Considering the fiber dimensions, we see that $\mathcal{V}$ and $\mathcal{U}$ are vector bundles of rank 2 and 1 , respectively. In fact, $s_{\Delta}$ induces an isomorphism $s_{\Delta}: \mathcal{O}_{T} \stackrel{\sim}{\longrightarrow} \mathcal{U}=p_{12 *} p_{23}^{*} \mathcal{O}(\Delta)$. Moreover, by Proposition 3.5 they fit into a short exact sequence

$$
0 \rightarrow \mathcal{V} \longrightarrow \mathcal{W} \stackrel{\rho}{\longrightarrow} \mathcal{O}_{T} \rightarrow 0
$$

Consequently, also $\mathcal{W}$ is a vector bundle and $\rho$ induces a map of geometric vector bundles

$$
\varrho: \underline{\operatorname{Spec}}_{T}\left(\operatorname{Sym}^{\bullet} \mathcal{W}^{\vee}\right) \longrightarrow T \times \mathbb{A}^{1}
$$

We set

$$
W:=\varrho^{-1}(T \times\{1\})
$$

with the projection $\tau: W \rightarrow T$. We retain some immediate consequences of the construction.

(i) $W$ is a principal homogeneous space under $\underline{\operatorname{Spec}}_{T}\left(\operatorname{Sym}^{\bullet} \mathcal{V}^{\vee}\right)$. In particular, it is an affine bundle over $T$.

(ii) Let $t=(\mathcal{L}, x) \in T$. Then by Lemma 3.6 we have

$$
W_{t}=\tau^{-1}(t) \cong \mathbb{P}\left(\operatorname{Ext}_{S}^{1}\left(i_{*} \mathcal{L}, i_{*}\left(\mathcal{L}(x) \otimes \omega_{C}^{-1}\right)\right)\right) \backslash \mathbb{P}\left(\operatorname{Ext}_{C}^{1}\left(\mathcal{L}, \mathcal{L}(x) \otimes \omega_{C}^{-1}\right)\right) .
$$

(iii) $\operatorname{dim} W=5$.

(vi) $W$ is compactified by the projective bundle $\bar{W}:=\mathbb{P}(\mathcal{W})$ with boundary isomorphic to $\mathbb{P}(\mathcal{V})$, i.e.

$$
\bar{W}=W \cup \mathbb{P}(\mathcal{V})
$$

Remark 3.7 Actually, $\mathcal{V} \cong p_{2}^{*}\left(\omega_{C} \oplus \omega_{C}\right)$ and hence $\mathbb{P}(\mathcal{V}) \cong \mathbb{P}^{1} \times \operatorname{Pic}^{1}(C) \times C$.

Next, we relate $E_{1}$ and $\bar{W}$. Recall that $N_{1}:=\bar{E}_{1} \subset N_{\text {red }}$. We keep all the notations from the previous section, and

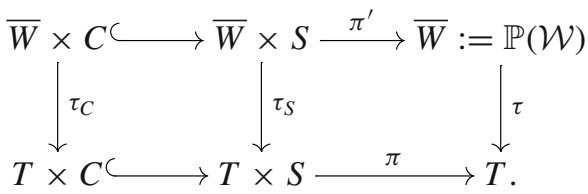

Proposition 3.8 There exists a 'universal' extension represented by

$0 \rightarrow \tau_{S}^{*}(\mathrm{id} \times i)_{*}\left(\mathcal{P}_{1} \otimes \mathcal{O}(\Delta) \otimes \omega_{C}^{-1}\right) \otimes \mathcal{O}_{\tau}(1) \rightarrow \mathcal{G}_{\text {univ }} \rightarrow \tau_{S}^{*}(\mathrm{id} \times i)_{*} p_{13}^{*} \mathcal{P}_{1} \rightarrow 0$, 
such that $\mathcal{G}_{\text {univ }} \in \operatorname{Coh}(\bar{W} \times S)$ defines a birational morphism

$$
v: \bar{W} \longrightarrow N_{1}
$$

In particular, $N_{\text {red }}=N_{0} \cup N_{1}$ is a decomposition into irreducible components.

Proof We set $\mathcal{F}:=\mathcal{P}_{1} \otimes \mathcal{O}(\Delta) \otimes \omega_{C}^{-1}$ and $\mathcal{F}^{\prime}:=p_{13}^{*} \mathcal{P}_{1}$. We are looking for a 'universal' extension, i.e. for

$$
v_{\text {univ }} \in \operatorname{Ext} \frac{1}{W \times S}\left(\tau_{S}^{*}(\mathrm{id} \times i)_{*} \mathcal{F}^{\prime}, \tau_{S}^{*}(\mathrm{id} \times i)_{*} \mathcal{F} \otimes \pi^{\prime *} \mathcal{O}_{\tau}(1)\right),
$$

such that for $w \in W \subset \bar{W}$ the restriction of $v_{\text {univ }}$ to $\{w\} \times S$ is the extension corresponding to $w \in W_{\tau(w)} \subset \operatorname{Ext}_{S}^{1}\left(i_{*} \mathcal{F}_{\tau(w)}^{\prime}, i_{*} \mathcal{F}_{\tau(w)}\right)$.

By definition, $\mathcal{W}=R^{1} \pi_{*} R \mathcal{H} \operatorname{om}\left((\mathrm{id} \times i)_{*} \mathcal{F}^{\prime},(\mathrm{id} \times i)_{*} \mathcal{F}\right)$. Hence, there is a base change map

$$
\tau^{*} \mathcal{W} \rightarrow R^{1} \pi^{\prime}{ }_{*} L \tau_{S}^{*} R \mathcal{H} \operatorname{om}\left((\mathrm{id} \times i)_{*} \mathcal{F}^{\prime},(\mathrm{id} \times i)_{*} \mathcal{F}\right)
$$

We get

$$
\begin{aligned}
& H^{0}\left(\bar{W}, \tau^{*} \mathcal{W} \otimes \mathcal{O}_{\tau}(1)\right) \rightarrow H^{0}\left(\bar{W}, R^{1} \pi^{\prime}{ }_{*} L \tau_{S}^{*} R \mathcal{H} \operatorname{Hom}\left((\mathrm{id} \times i)_{*} \mathcal{F}^{\prime},(\mathrm{id} \times i)_{*} \mathcal{F}\right) \otimes \mathcal{O}_{\tau}(1)\right) \\
& \left.\quad \sim H^{1}\left(\bar{W} \times S, L \tau_{S}^{*} R \mathcal{H} \operatorname{Hom}\left((\mathrm{id} \times i)_{*} \mathcal{F}^{\prime},(\operatorname{id} \times i)_{*} \mathcal{F}\right) \otimes \pi^{\prime *} \mathcal{O}_{\tau}(1)\right)\right) \\
& \quad=\operatorname{Ext}_{\bar{W} \times S}^{1}\left(\tau_{S}^{*}(\operatorname{id} \times i)_{*} \mathcal{F}^{\prime}, \tau_{S}^{*}(\operatorname{id} \times i)_{*} \mathcal{F} \otimes \pi^{\prime *} \mathcal{O}_{\tau}(1)\right),
\end{aligned}
$$

where the indicated isomorphism comes from the Leray spectral sequence. It is an isomorphism, because

$$
\begin{aligned}
& R^{0} \pi^{\prime}{ }_{*} L \tau_{S}^{*} R \mathcal{H} \operatorname{Hom}\left((\mathrm{id} \times i)_{*} \mathcal{F}^{\prime},(\mathrm{id} \times i)_{*} \mathcal{F}\right) \\
& \cong R^{0} \pi_{C *}^{\prime} L \tau_{C}^{*} R \mathcal{H} \operatorname{Hom}\left(L(\mathrm{id} \times i)^{*}(\mathrm{id} \times i)_{*} \mathcal{F}^{\prime}, \mathcal{F}\right)=0,
\end{aligned}
$$

where $\pi_{C}: T \times C \rightarrow T$. The last equality follows from the long exact sequence

$$
\begin{aligned}
\cdots \rightarrow 0 & =R^{0} \pi_{C *}^{\prime} L \tau_{C}^{*} R \mathcal{H} \operatorname{Hom}\left(\mathcal{F}^{\prime}, \mathcal{F}\right) \rightarrow R^{0} \pi_{C *}^{\prime} L \tau_{C}^{*} R \mathcal{H o m}\left(L(\mathrm{id} \times i)^{*}(\mathrm{id} \times i)_{*} \mathcal{F}^{\prime}, \mathcal{F}\right) \\
\rightarrow 0 & =R^{0} \pi_{C *}^{\prime} L \tau_{C}^{*} R \mathcal{H} \operatorname{Hom}\left(\mathcal{F}^{\prime} \otimes \mathcal{O}_{C}(-C)[1], \mathcal{F}\right) \rightarrow \ldots
\end{aligned}
$$

Finally, we consider the universal surjection as an element in $\left.H^{0}\left(\bar{W}, \tau^{*} \mathcal{W} \otimes \mathcal{O}_{\tau}(1)\right)\right)$ and take its image under (3.7). This produces the desired extension.

By construction, $\mathcal{G}_{\text {univ }} \in \operatorname{Coh}(\bar{W} \times S)$ defines a morphism $v: \bar{W} \rightarrow N_{1} \subset M$ which restricts to a bijection $W \rightarrow E_{1}$ (see Corollary 3.4 and (3.3)). By degree reasons an extension on $C$ of the form $0 \rightarrow \mathcal{L} \rightarrow \mathcal{E} \rightarrow \mathcal{L}^{\prime} \rightarrow 0$, where $\operatorname{deg} \mathcal{L}^{\prime}=1$ and $\operatorname{deg} \mathcal{L}=0$ is stable or split. However, the split extensions do not occur in $\mathbb{P}(\mathcal{V})$. Hence, $v$ is everywhere defined. Moreover, the boundary $\bar{W} \backslash W=\mathbb{P}(\mathcal{V})$ maps to $N_{1} \backslash E_{1}=N_{0} \cap N_{1}$. 
Remark 3.9 One can show that $v: W \rightarrow E_{1}$ is actually an isomorphism of schemes. Moreover, $v: \bar{W} \rightarrow N_{1}$ is finite and hence a normalization map. Its tangent map is analyzed in [11, Proposition 7.5] and provides a characterization of the singularities of $N_{1}$.

\section{Proof of Theorem 1.1}

We will now prove Theorem 1.1.

Theorem Let $C \in|H|$ be an irreducible curve. The degrees of the two components of the nilpotent cone $N_{C}=N_{0} \cup N_{1}$ are given by

$$
\operatorname{deg}_{u_{1}} N_{0}=5 \cdot 2^{9} \text { and } \operatorname{deg}_{u_{1}} N_{1}=5^{2} \cdot 2^{11}
$$

and their multiplicities are

$$
\text { mult }_{N_{C}} N_{0}=2^{3} \text { and } \text { mult }_{N_{C}} N_{1}=2 \text {. }
$$

Moreover, any fiber $F$ of the Mukai system has degree $5 \cdot 3 \cdot 2^{13}$.

All degrees will be computed with respect to a naturally defined distinguished ample class $u_{1} \in H^{2}(M, \mathbb{Z})$, which we construct in Sect. 4.1. We set

$$
d_{i}=\operatorname{deg}_{u_{1}}\left(N_{i}\right):=\int_{M}\left[N_{i}\right] u_{1}^{5}
$$

for $i=0,1$, where by abuse of notation $\left[N_{i}\right] \in H^{10}(M, \mathbb{Z})$ is the Poincaré dual of the fundamental homology class $\left[N_{i}\right] \in H_{10}(M, \mathbb{Z})$.

The multiplicity is defined as follows. Let $\eta_{i}$ be the generic point of $N_{i}$. Then

$$
m_{i}=\operatorname{mult}_{N} N_{i}:=\lg _{\mathcal{O}_{N, \eta_{i}}} \mathcal{O}_{N_{i}, \eta_{i}}=\lg _{\mathcal{O}_{N_{i}, \eta_{i}}} \mathcal{O}_{N_{i}, \eta_{i}}
$$

In particular, we have an equality $[F]=m_{0}\left[N_{0}\right]+m_{1}\left[N_{1}\right] \in H^{10}(M, \mathbb{Z})$ for any fiber $F$. Consequently, inserting $m_{0}=2^{3}$ and $m_{1}=2$, we find

$$
\operatorname{deg}_{u_{1}}(F)=5 \cdot 2^{12}+5^{2} \cdot 2^{12}=5 \cdot 3 \cdot 2^{13} .
$$

as stated in the theorem. Luckily, it turns out that the multiplicities are small in comparison with the degrees so that it is possible to determine the multiplicities from the knowledge of the degrees but not vice versa.

Proof of the multiplicities knowing all the degrees Let $F \subset M$ be a smooth fiber. Then, we have $\operatorname{deg} F=m_{0} d_{0}+m_{1} d_{1}$ and hence

$$
5 \cdot 3 \cdot 2^{13}=m_{0} \cdot 5 \cdot 2^{9}+m_{1} \cdot 5^{2} \cdot 2^{11}
$$


The only possible solutions are $\left(m_{0}, m_{1}\right)=(28,1)$ or $\left(m_{0}, m_{1}\right)=(8,2)$. However, by [8, Proposition 4.11]

$$
\operatorname{dim} T_{[\mathcal{E}]} N=\operatorname{dim} \operatorname{Ext}_{2 C}^{1}(\mathcal{E}, \mathcal{E})=\operatorname{dim} N+1 \text { for all }[\mathcal{E}] \in E_{1} .
$$

Hence, $N_{1}$ is not reduced and the first solution is ruled out.

Remark 4.1 We will prove Theorem 1.1 for a fixed smooth curve $C \in|H|$, which implies the case of an irreducible and possibly singular curve by a deformation argument as follows. According to the careful analysis in [6, Section 3.7, in particular Propositions 3.7.23 \& 3.7.19] the above description of the irreducible components of $f^{-1}(2 C)$ is valid for every irreducible curve $C \in|H|$. Hence, if one deforms from a smooth to a singular, irreducible curve in $|H|$, the irreducible components of the fiber with their reduced structure deform as well. Consequently, degrees and multiplicities remain constant.

\subsection{Construction of the ample class $u_{1}$}

We use the determinant line bundle construction [16, Lemma 8.1.2] in order to produce an ample class on the moduli space $M$.

Let $X$ and $T$ be two projective varieties and assume that $X$ is smooth. Let $p: T \times$ $X \rightarrow T$ and $q: T \times X \rightarrow X$ denote the two projections. For any $\mathcal{W} \in \operatorname{Coh}(X \times T)$ flat over $T$, we define $\lambda_{\mathcal{W}}: \mathrm{K}(X)_{\text {num }} \rightarrow H^{2}(T, \mathbb{Z})$ to be the following composition

$$
\mathrm{K}(X)_{\text {num }} \stackrel{q^{*}}{\rightarrow} \mathrm{K}^{0}(T \times X)_{\text {num }} \stackrel{[\mathcal{W}]}{\longrightarrow} \mathrm{K}^{0}(T \times X)_{\text {num }} \stackrel{R p_{*}}{\longrightarrow} \mathrm{K}^{0}(T)_{\text {num }} \stackrel{\text { det }}{\longrightarrow} \mathrm{NS}(T) \subset H^{2}(T, \mathbb{Z}) .
$$

We will take advantage of the functorial properties of this definition. These are

(i) $f^{*} \lambda_{\mathcal{W}}=\lambda_{(f \times \text { id }) * \mathcal{W}}$ for any morphism $f: T^{\prime} \rightarrow T$ and

(ii) $\lambda_{(\mathrm{id} \times i)_{*} \mathcal{W}}(x)=\lambda_{\mathcal{W}}\left(L i^{*} x\right)$ for all $x \in \mathrm{K}(X)_{\text {num }}$ if $i: Y \hookrightarrow X$ is the inclusion of a closed, smooth subscheme and $\mathcal{W} \in \operatorname{Coh}(T \times Y)$.

The construction is especially interesting if $X=\mathrm{M}_{T}(c)$ is a fine moduli space, that parametrizes coherent sheaves of class $c$ on $T$. Let $\mathcal{E}_{\text {univ }}$ be a universal sheaf on $\mathrm{M}_{T}(c) \times T$, then

$$
\lambda \mathcal{E}_{\text {univ }} \otimes p^{*} \mathcal{M}(x)=\lambda \mathcal{E}_{\text {univ }}(x)+\chi(c \cdot x) c_{1}(\mathcal{M})
$$

for all $\mathcal{M} \in \operatorname{Pic}\left(\mathrm{M}_{T}(c)\right)$. Hence,

$$
\lambda_{\mathrm{M}_{T}(c)}:=\lambda_{\mathcal{E}_{\text {univ }}}: c^{\perp, \chi} \longrightarrow \mathrm{NS}\left(\mathrm{M}_{T}(c)\right)
$$

is well-defined and does not depend on the choice of universal sheaf. Here,

$$
c^{\perp, \chi}=\left\{x \in \mathrm{K}(T)_{\text {num }} \mid \chi(x \cdot c)=0\right\} .
$$


Example 4.2 Let $C$ be a smooth curve of any genus $g \geq 0$. Then

$$
\left(\text { rk, deg) : } \mathrm{K}(C)_{\text {num }} \stackrel{\sim}{\longrightarrow} \mathbb{Z} \oplus \mathbb{Z}\right. \text {. }
$$

Fix $n \geq 1$ and $d \in \mathbb{Z}$ coprime and let $c=(n, d) \in \mathrm{K}(C)_{\text {num. }}$. Then $\mathrm{M}_{C}(c)=\mathrm{M}_{C}(n, d)$ is the moduli space of stable vector bundles of rank $n$ and degree $d$ on $C$ and we find $c^{\perp, \chi}=\langle(-n, d+n(1-g)\rangle$. The generalized Theta divisor can be defined by

$$
\Theta_{\mathrm{M}_{C}(n, d)}:=\lambda_{\mathrm{M}_{C}(n, d)}(-n, d+n(1-g)),
$$

see [12, Théorème D]. A special case is $\mathrm{M}_{C}(1, k)=\operatorname{Pic}^{k}(C)$. In this case, we find $c^{\perp, \chi}=\langle(-1, k+1-g)\rangle$ and

$$
\Theta_{k}:=\lambda_{\operatorname{Pic}^{k}(C)}(-1, k+1-g)
$$

is the class of the canonical Theta divisor in $\operatorname{Pic}^{k}(C)$.

Remark 4.3 Denote by $\operatorname{SM}_{C}(n, d)$ the moduli space of vector bundles with fixed determinant, i.e. a fiber of det: $\operatorname{M}_{C}(n, d) \rightarrow \operatorname{Pic}(C)$ and by $\Theta_{\mathrm{SM}_{C}(n, d)}$ the restriction of $\Theta_{\mathrm{M}_{C}(n, d)}$ to $\mathrm{SM}_{C}(n, d)$. Taking the tensor product defines an étale map

$$
h: \operatorname{SM}_{C}(n, d) \times \operatorname{Pic}^{0}(C) \longrightarrow \mathrm{M}_{C}(n, d)
$$

of degree $n^{2 g}$. Using [10, Corollary 6], we find the following relation if $(n, d)$ are coprime

$$
h^{*} \Theta_{\mathrm{M}_{C}(n, d)}=p_{1}^{*} \Theta_{\mathrm{SM}_{C}(n, d)}+n^{2} p_{2}^{*} \Theta_{0} .
$$

Lemma 4.4 Let $C$ be a smooth curve of genus $g$ and $\mathcal{P}$ a Poincaré line bundle on $\operatorname{Pic}^{k}(C) \times C$. Then

$$
\lambda_{\mathcal{P}}: \mathrm{K}(C)_{\text {num }} \rightarrow H^{2}\left(\operatorname{Pic}^{k}(C), \mathbb{Z}\right)
$$

is given by

$$
(r, d) \mapsto(d+(k+1-g) r) \mu-r \Theta_{k},
$$

where $p_{1}^{*} \mu=c_{1}^{2,0}(\mathcal{P}) \in H^{2}\left(\operatorname{Pic}^{k}(C) \times C, \mathbb{Z}\right)$ is the $(2,0)$ Künneth component of $c_{1}(\mathcal{P})$.

By tensoring with a suitable line bundle on $\operatorname{Pic}^{k}(C)$, we can assume $c_{1}^{2,0}(\mathcal{P})=0$.

Proof Let us abbreviate $\mathrm{Pic}^{k}(C)$ to $\mathrm{Pic}^{k}$. We decompose

$$
c_{1}(\mathcal{P})=c^{2,0}+c^{1,1}+c^{0,2}
$$

into its Künneth components and write $c^{2,0}=p^{*} \mu$ for some $\mu \in H^{2}\left(\mathrm{Pic}^{k}, \mathbb{Z}\right)$. Then by [1, VIII §2] the class $\gamma=c^{1,1}$ satisfies $\gamma^{2}=-2 \rho p^{*} \Theta_{k}$. Moreover, by 
definition, $c^{0,2}=k \rho$, where $\rho$ is the pullback of the class of a point on $C$. Together, $c_{1}(\mathcal{P})=p^{*} \mu+\gamma+k \rho$ and

$$
\operatorname{ch}(\mathcal{P})=1+p^{*} \mu+\gamma+k \rho+\rho p^{*}\left(k \mu-\Theta_{k}\right) .
$$

Now, let $x=(r, d) \in \mathrm{K}(C)_{\text {num. }}$. The Grothendieck-Riemann-Roch theorem gives

$$
\begin{aligned}
\operatorname{ch}\left(R p_{*}\left(\mathcal{P} \otimes q^{*} x\right)\right) & =p_{*}\left(\operatorname{ch}\left(\mathcal{P} \otimes q^{*} x\right) \operatorname{td}\left(\operatorname{Pic}^{k} \times C\right)\right)=p_{*}\left(\operatorname{ch}(\mathcal{P}) \operatorname{ch}\left(q^{*} x\right) q^{*} \operatorname{td}(C)\right) \\
& =p_{*}(\operatorname{ch}(\mathcal{P})(r+((1-g) r+d) \rho)) \\
& =k r+(1-g) r+d+(k r+(1-g) r)+d) \mu-r \Theta_{k}
\end{aligned}
$$

In particular, $\lambda_{\mathcal{P}}(x)=c_{1}\left(R p_{*}\left(\mathcal{P} \otimes q^{*} x\right)\right)=((k+1-g) r+d) \mu-r \Theta_{k}$.

We come back to our original situation, i.e. $(S, H)$ is a polarized $\mathrm{K} 3$ surface of genus 2 and $M=M_{H}(v)$ parametrizes $H$-stable sheaves with fixed Mukai vector $v=(0,2 H,-1)$ or equivalently, with Chern character $v_{\mathrm{ch}}=(0,2 H,-1)$. In this setting $\lambda_{M}$ induces an isomorphism, [16, Theorem 6.2.15]

$$
\lambda_{M}: v_{\mathrm{ch}}^{\perp, \chi} \stackrel{\sim}{\longrightarrow} \mathrm{NS}(M)
$$

As $v$ and $v_{\text {ch }}$ coincide, we will notationally not distinguish between them anymore. We find

$$
v^{\perp, \chi}=\{(2 c . H, c, s) \mid c \in \mathrm{NS}(S), s \in \mathbb{Z}\} .
$$

Warning 4.5 In this setting, one usually wants to consider the morphism $\lambda_{M}$ in terms of the Mukai vector and the Mukai pairing instead of the chern character and the intersection product, i.e. one considers the composition

$$
v^{\perp,\langle,\rangle} \stackrel{\sim}{\longrightarrow} v_{\mathrm{ch}}^{\perp, \chi} \stackrel{\lambda_{M}}{\longrightarrow} \mathrm{NS}\left(M_{H}(v)\right),
$$

which identifies the Mukai pairing on the left hand side with the Beauville-Bogomolov form on the right hand side. Here $v_{\mathrm{ch}} \cdot \sqrt{\operatorname{td}(S)}=v$. Explicitly, if $v=(r, c, s)$, then $v_{\mathrm{ch}}=(r, c, s-r)$ and

$$
\left\langle\left(r^{\prime}, c^{\prime}, s^{\prime}\right),(r, c, s)\right\rangle=\chi\left(\left(-r^{\prime}, c^{\prime},-s^{\prime}-r^{\prime}\right) \cdot(r, c, s-r)\right) .
$$

Thus the first arrow is given by $\left(r^{\prime}, c^{\prime}, s^{\prime}\right) \mapsto\left(-r^{\prime}, c^{\prime},-s^{\prime}-r^{\prime}\right)$.

Definition 4.6 For all $s \in \mathbb{Z}$ we define

$$
l_{s}:=\lambda_{M}((-4,-H, s)) \in H^{2}(M, \mathbb{Z})
$$


The value of $s$ does not have any relevance for our computations. However, with the results of [3], it can be proven that $l_{s}$ is ample for $s \gg 0$ and one can even compute the precise boundary of the ample cone.

Definition 4.7 For everything what follows, we fix $s_{0} \gg 0$ such that $l_{s_{0}}$ is ample and set

$$
u_{1}:=l_{s_{0}} .
$$

\subsection{Degree of a general fiber}

We compute the degree of a general fiber.

Proposition 4.8 Let $D \in|2 H|$ be a smooth curve and let $F:=f^{-1}(D)$ be the corresponding fiber. Let $u=\lambda_{M}(x)$ with $x=(2 c . H, c, s) \in v^{\perp, \chi}$. Then

$$
\left.u\right|_{F}=-2 c . H \cdot \Theta_{3},
$$

where $\Theta_{3} \in H^{2}\left(\operatorname{Pic}^{3}(D), \mathbb{Z}\right)$ is the class of the Theta divisor. In particular, we have

$$
\operatorname{deg}_{u_{1}} F=5 ! \cdot 2^{10}
$$

Proof Let $i: D \hookrightarrow S$ be the inclusion. The inclusion $\operatorname{Pic}^{3}(D) \cong F \hookrightarrow M$ is defined by $(\mathrm{id} \times i)_{*} \mathcal{P}_{3}$, where $\mathcal{P}_{3}$ is a Poincaré line bundle on $\operatorname{Pic}^{3}(D) \times D$. Hence,

$$
\left.u\right|_{\operatorname{Pic}^{3}(D)}=\lambda_{(\mathrm{id} \times i)_{*} \mathcal{P}_{3}}(x)=\lambda_{\mathcal{P}_{3}}\left(L i^{*} x\right) .
$$

Now, $L i^{*}: K(S)_{\text {num }} \rightarrow K(D)_{\text {num }} \cong \mathbb{Z}^{\oplus 2}$ maps $(r, c, s)$ to $(r, c . D)$ and thus $L i^{*} x$ to $2 c . H \cdot(1,1)$, whereas by definition $\theta_{3}=\lambda_{\mathcal{P}_{3}}(-1,-1)$. Finally,

$$
\operatorname{deg}_{u_{1}} F=\int_{\operatorname{Pic}^{3}(D)}\left(4 \Theta_{3}\right)^{5}=2^{10} \cdot 5 !
$$

Remark 4.9 One can also prove the above result using the Beauville-Bogomolov form $(,)_{B B}$ on $H^{2}(M, \mathbb{Z})$. Let $u_{0}=f^{*} c_{1}(\mathcal{O}(1)) \in H^{2}(M, \mathbb{Z})$. Then $[F]=u_{0}^{5} \in$ $H^{10}(M, \mathbb{Z})$ and

$$
\operatorname{deg}_{u_{1}}(F)=\int_{M} u_{0}^{5} u_{1}^{5}=5 ! \cdot\left(u_{0}, u_{1}\right)_{B B}^{5},
$$

where we use that $\left(u_{0}, u_{0}\right)_{B B}=0$ and that $M$ is birational to $S^{[5]}$ in order to determine the correct Fujiki constant. One verifies that $u_{0}=\lambda_{M}((0,0,1))$ [25, Lem 4.4] whereas, by definition, $u_{1}=\lambda_{M}\left(-4,-H, s_{0}\right)$ with $s_{0} \gg 0$. After correct identification (cf. Warning 4.5), one has

$$
\left(\lambda_{M}(r, c, s), \lambda_{M}\left(r^{\prime}, c^{\prime}, s^{\prime}\right)\right)_{B B}=\left\langle(r, c, s),\left(r^{\prime}, c^{\prime}, s^{\prime}\right)\right\rangle+2 r r^{\prime} .
$$


This gives $\left(u_{0}, u_{1}\right)_{B B}=4$.

\subsection{Degree of the vector bundle component $N_{0}$}

Next, we deal with the component $N_{0}$, which is isomorphic to $M_{C}(2,1)$.

Proposition 4.10 Let $x=\lambda_{M}(u)$ with $u=(2 c . H, c, s) \in v^{\perp, \chi}$. Then

$$
\left.x\right|_{N_{0}}=-c . H \Theta,
$$

where $\Theta \in H^{2}\left(N_{0}, \mathbb{Z}\right)$ is the the generalized Theta divisor. In particular,

$$
\left.u_{1}\right|_{N_{0}}=2 \Theta,
$$

and given $x_{i}=\lambda_{M}\left(2 c_{i} . H, c_{i}, s_{i}\right)$ for $i=1, \ldots, 5$, we find

$$
\int_{M} x_{1} \ldots x_{5}\left[N_{0}\right]=-\prod_{i=1}^{5} c_{i} . H \int_{N_{0}} \Theta^{5}=-5 \cdot 2^{4} \prod_{i=1}^{5} c_{i} . H .
$$

Hence, $\operatorname{deg}_{u_{1}} N_{0}=5 \cdot 2^{9}$.

Proof Let $i: C \hookrightarrow S$ be the inclusion. The inclusion $N_{0} \hookrightarrow M$ is defined by $(\mathrm{id} \times i)_{*} \mathcal{E}_{\text {univ }}$, where $\mathcal{E}_{\text {univ }}$ is the universal vector bundle on $N_{0} \times C$. Hence,

$$
\left.x\right|_{N_{0}}=\lambda_{(\mathrm{id} \times i)_{*} \mathcal{E}_{\text {univ }}}(u)=\lambda_{N_{0}}\left(L i^{*} u\right) .
$$

Now, $L i^{*}: K(S)_{\text {num }} \rightarrow K(C)_{\text {num }} \cong \mathbb{Z}^{\oplus 2}$ maps $(r, c, s)$ to $(r, c . H)$. In particular, $L i^{*} u=c . H(2,1)$, whereas by definition $\theta=\lambda_{N_{0}}(-2,-1)$.

Next, we compute $\int_{N_{0}} \Theta^{5}$ by pulling back along $h: \operatorname{SM}_{C}(2,1) \times \operatorname{Pic}^{0}(C) \rightarrow N_{0}$ from Remark 4.3.

$$
\begin{aligned}
\int_{\mathrm{M}_{C}(2,1)} \Theta^{5} \stackrel{(4.1)}{=} \frac{1}{2^{4}} \int_{\mathrm{SM}_{C}(2,1) \times \operatorname{Pic}^{0}(C)}\left(p_{1}^{*} \Theta_{\mathrm{SM}}+4 p_{2}^{*} \Theta_{0}\right)^{5} \\
=\frac{1}{2^{4}}\left(\begin{array}{l}
5 \\
3
\end{array}\right) \int_{\mathrm{SM}_{C}(2,1)} \Theta_{\mathrm{SM}}^{3} \int_{\operatorname{Pic}^{0}(C)}\left(4 \Theta_{0}\right)^{2}=5 \cdot 2^{4}
\end{aligned}
$$
[27].

The value $\int_{\mathrm{SM}_{C}(2,1)} \Theta_{\mathrm{SM}}^{3}=4$ is given by the leading term of the Verlinde formula

Remark 4.11 The general formula is

$$
\int_{\mathrm{M}_{C}(n, d)} \Theta^{\operatorname{dim} \mathrm{M}_{C}(n, d)}=\operatorname{dim} \mathrm{M}_{C}(n, d) !\left(2^{2 g-2}-2\right) \frac{(-1)^{g} 2^{2 g-2} B_{2 g-2}}{(2 g-2) !},
$$

where $B_{i}$ is the $i$-th Bernoulli number. The second Bernoulli number is $B_{2}=\frac{1}{6}$ 
Remark 4.12 In the general case, where $\left[v=(0, n H, s)\right.$ and $u_{1}=\lambda_{M}(-n(2 g-$ 2), $s H, *)$ with $s=n+d(1-g)$, ] we find

$\left.u_{1}\right|_{F}=n(2 g-2) \Theta_{\delta}$ and $\left.u_{1}\right|_{N_{0}}=(2 g-2) \Theta$. Thus

$$
\begin{aligned}
& \operatorname{deg}_{u_{1}} F=(n(2 g-2))^{\operatorname{dim} N} \cdot \operatorname{dim} N ! \\
& \text { and } \operatorname{deg}_{u_{1}} N_{0}=(2 g-2)^{\operatorname{dim} N} \int_{\mathrm{M}_{C}(n, d)} \Theta^{\operatorname{dim}^{\operatorname{dim}(n, d)} .}
\end{aligned}
$$

Here, $\operatorname{dim} N=n^{2}(2 g-2)+2$.

\subsection{Degree of the other component $N_{1}$}

We complete the proof of Theorem 1.1 by dealing with the remaining component $N_{1}$. Recall from Proposition 3.8 that there is a birational map $v: \bar{W} \rightarrow N_{1}$, where $\tau: \bar{W}=\mathbb{P}(\mathcal{W}) \rightarrow T=\operatorname{Pic}^{1}(C) \times C$.

Proposition 4.13 Let $x_{i}=\lambda_{M}\left(u_{i}\right)$ with $u_{i}=\left(2 c_{i} . H, c_{i}, s_{i}\right) \in v^{\perp, \chi}$ for $i=1, \ldots, 5$. Then

$$
\int_{M} x_{1} \ldots x_{5}\left[N_{1}\right]=\int_{\bar{W}} \prod_{i=1}^{5} v^{*}\left(\left.x_{i}\right|_{N_{1}}\right)=-5^{2} \cdot 2^{6} \prod_{i=1}^{5} c_{i} . H .
$$

In particular, $\operatorname{deg}_{u_{1}} N_{1}=5^{2} \cdot 2^{11}$.

Note that the first equality in (4.3) is immediate, because $v: \bar{W} \rightarrow N_{1}$ is birational. For the proof of the proposition, we need to introduce some more notation. We abbreviate $\operatorname{Pic}^{1}(C)$ to $\mathrm{Pic}^{1}$ and in the following all cohomology groups have $\mathbb{Z}$ coefficients. We set

$$
\zeta=c_{1}\left(\mathcal{O}_{\tau}(1)\right) \in H^{2}(\bar{W}) \text { and write } \rho=p_{2}^{*}[\mathrm{pt}] \in \mathrm{H}^{2}\left(\mathrm{Pic}^{1} \times \mathrm{C}\right)
$$

for the pullback of the class of a point on $C$. If no confusion is likely, we suppress pullbacks from our notation, e.g. we will write $\Theta_{1} \in H^{2}\left(\operatorname{Pic}^{1} \times C\right)$ and also $\Theta_{1} \in$ $H^{2}(\mathbb{P}(\mathcal{W}))$ instead of $p_{1}^{*} \Theta_{1}$ and $\tau^{*} p_{1}^{*} \Theta_{1}$, respectively. Moreover, we define

$$
\pi:=c_{1}(\mathcal{P})-c_{1}^{2,0}(\mathcal{P}) \in H^{2}\left(\operatorname{Pic}^{1} \times C\right),
$$

where $\mathcal{P}$ is a Poincaré line bundle. Note that $\pi$ is independent of the choice of $\mathcal{P}$.

Proof of Proposition 4.13 We will split the proof into the following three steps.

(i) Let $x=\lambda_{M}(2 c . H, c, s)$. Then

$$
v^{*}\left(\left.x\right|_{N_{1}}\right)=\lambda_{\mathcal{G}_{\text {univ }}}(x)=c . H\left(-4 \Theta_{1}+2 \pi-7 \rho-\zeta\right) \in H^{2}(\bar{W}) .
$$

(ii) We have

$$
\left(-4 \Theta_{1}+2 \pi-7 \rho-\zeta\right)^{5}=-5^{2} 2^{5} \zeta^{2} \rho \Theta_{1}^{2} \in H^{10}(\bar{W}) .
$$


(iii) The top cohomology group $H^{10}(\bar{W})$ generated by $\frac{1}{2} \zeta^{2} \rho \Theta_{1}^{2}$ and we have

$$
\int_{\mathbb{P}(\mathcal{W})} \zeta^{2} \rho \Theta_{1}^{2}=2
$$

Proof of (i) In Proposition 3.8, we defined the morphism $v: \bar{W} \rightarrow N_{1}$ by means of $\mathcal{G}_{\text {univ }} \in \operatorname{Coh}(\bar{W} \times S)$, which sits in the (universal) extension

$0 \rightarrow \tau_{S}^{*}(\mathrm{id} \times i)_{*}\left(\mathcal{P}_{1} \otimes \mathcal{O}(\Delta) \otimes \omega_{C}^{-1}\right) \otimes \mathcal{O}_{\tau}(1) \rightarrow \mathcal{G}_{\text {univ }} \rightarrow \tau_{S}^{*}(\mathrm{id} \times i)_{*} p_{13}^{*} \mathcal{P}_{1} \rightarrow 0$

where $\tau_{S}=\tau \times \operatorname{id}_{S}: \bar{W} \times S \rightarrow \operatorname{Pic}^{1} \times C \times S$. So, by construction, we have

$$
\begin{aligned}
\lambda_{\mathcal{G}_{\text {univ }}}(x) & =\lambda_{\tau_{S}^{*}(\operatorname{id} \times i)_{*}\left(\mathcal{P}_{1} \otimes \mathcal{O}(\Delta) \otimes \omega_{C}^{-1}\right) \otimes \mathcal{O}_{\tau}(1)}(x)+\lambda_{\tau_{S}^{*}(\mathrm{id} \times i)_{*} p_{13}^{*} \mathcal{P}_{1}}(x) \\
& =\lambda_{\tau_{S}^{*}(\mathrm{id} \times i)_{*}\left(\mathcal{P}_{1} \otimes \mathcal{O}(\Delta) \otimes \omega_{C}^{-1}\right)}(x)+k\left(L i^{*} x\right) \cdot \zeta+\tau^{*} p_{1}^{*} \lambda \mathcal{P}_{1}\left(L i^{*} x\right) \\
& =\tau^{*}\left(\lambda_{\mathcal{P}_{1} \otimes \mathcal{O}(\Delta)}\left(L i^{*} x \cdot \omega^{-1}\right)+p_{1}^{*} \lambda_{\mathcal{P}_{1}}\left(L i^{*} x\right)\right)+k\left(L i^{*} x\right) \cdot \zeta,
\end{aligned}
$$

where

$\omega=c_{1}\left(\omega_{C}\right)$ and $k\left(L i^{*} x\right)=\operatorname{rk} R p_{*}\left(\mathcal{P}_{1} \otimes \mathcal{O}(\Delta) \otimes \omega_{C}^{-1} \otimes L i^{*} x\right)=\chi\left(L i^{*} x\right)=-c . H$.

The term $\lambda_{\mathcal{P}_{1} \otimes \mathcal{O}(\Delta)}\left(L i^{*} x \cdot \omega^{-1}\right)+p_{1}^{*} \lambda_{\mathcal{P}_{1}}\left(L i^{*} x\right)$, is determined in Lemmas 4.14 and 4.4. Note that each summand depends on the choice of a Poincaré line bundle, whereas the sum does not. Together,

$$
\begin{aligned}
v^{*}\left(\left.x\right|_{N_{1}}\right) & =\tau^{*}\left(\lambda_{\mathcal{P}_{1} \otimes \mathcal{O}(\Delta)}\left(L i^{*} x \cdot \omega^{-1}\right)+p_{1}^{*} \lambda_{\mathcal{P}_{1}}\left(L i^{*} x\right)\right)-c . H \zeta \\
& =c . H\left(p_{1}^{*}\left(\lambda_{\mathcal{P}_{1}}(2,-3)+\lambda_{\mathcal{P}_{1}}(2,1)\right)+2 c_{1}\left(\mathcal{P}_{1}\right)-7 \rho-\zeta\right) \\
& =c . H\left(-4 \Theta_{1}+2 \pi-7 \rho-\zeta\right) .
\end{aligned}
$$

Lemma 4.14 Let $\mathcal{F} \in \operatorname{Coh}(X \times C)$. Then

$$
\lambda_{\mathcal{F} \otimes \mathcal{O}(\Delta)}(x)=p_{1}{ }^{*} \lambda_{\mathcal{F}}(x)+r c_{1}(\mathcal{F})+c_{0}(\mathcal{F})(d-2 r) \rho
$$

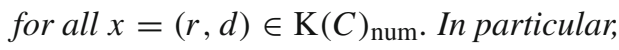

$$
\lambda_{\mathcal{P}_{1} \otimes \mathcal{O}(\Delta)}\left(L i^{*} x \cdot \omega^{-1}\right)=c . H\left(p_{1}^{*} \lambda_{\mathcal{P}}(2,-3)+2 c_{1}\left(\mathcal{P}_{1}\right)-7 \rho\right) .
$$

Proof We have

$$
[\mathcal{F} \otimes \mathcal{O}(\Delta)]=\left[p_{13}^{*} \mathcal{F}\right]+\left[\left(\mathrm{id} \times i_{\Delta}\right)_{*}\left(p_{2}^{*} \omega_{C}^{-1} \otimes \mathcal{F}\right)\right] \in \mathrm{K}(X \times C \times C)
$$

where $i_{\Delta}: C \rightarrow C \times C$ is the diagonal and thus

$$
\lambda_{\mathcal{F} \otimes \mathcal{O}(\Delta)}(x)=\lambda_{p_{13}^{*} \mathcal{W}}(x)+\lambda_{\left(\mathrm{id} \times i_{\Delta}\right)_{*}\left(p_{2}^{*} \omega_{C}^{-1} \otimes \mathcal{F}\right)}(x) \in H^{2}(X \times C)
$$


for all $x \in \mathrm{K}(C)_{\text {num. Now, }}$.

$$
\begin{aligned}
\lambda_{\left(\mathrm{id} \times i_{\Delta}\right)_{*}\left(p_{2}^{*} \omega^{-1} \cdot[\mathcal{F}]\right)}(x) & =\operatorname{det} R p_{12 *}\left(\left(\mathrm{id} \times i_{\Delta}\right)_{*}\left(p_{2}^{*} \omega^{-1} \cdot[\mathcal{F}]\right) \cdot p_{3}^{*} x\right) \\
& =\operatorname{det} R\left(p_{12} \circ\left(\mathrm{id} \times i_{\Delta}\right)\right)_{*}\left([\mathcal{F}] \cdot p_{2}^{*}\left(\omega^{-1} \cdot x\right)\right) \\
& =\operatorname{det}\left([\mathcal{F}] \cdot p_{2}^{*}\left(\omega^{-1} \cdot x\right)\right)=r c_{1}(\mathcal{F})+c_{0}(\mathcal{F})(d-r(2 g-2)) \rho .
\end{aligned}
$$

To prove the remaining steps, we need to understand the cohomology ring $H^{*}(\bar{W})$.

\section{Lemma 4.15 We have}

$$
H^{*}(\bar{W}) \cong H^{*}\left(\operatorname{Pic}^{1} \times C\right)[\zeta] / \zeta^{3}+4 \rho \zeta^{2}
$$

In particular,

$$
H^{10}(\bar{W})=\zeta^{2} \cdot H^{6}\left(\operatorname{Pic}^{1} \times C\right)
$$

Proof By definition, $\bar{W}=\mathbb{P}(\mathcal{W})$. Hence,

$$
H^{*}(\bar{W}) \cong H^{*}\left(\operatorname{Pic}^{1} \times C\right)[\zeta] / \zeta^{3}+c_{1}(\mathcal{W}) \zeta^{2}+c_{2}(\mathcal{W}) \zeta+c_{3}(\mathcal{W})
$$

We use the short exact sequence $0 \rightarrow \mathcal{V} \rightarrow \mathcal{W} \rightarrow \mathcal{O}_{T} \rightarrow 0$ from (3.6) to compute the Chern classes of $\mathcal{W}$. Note that

$$
\mathcal{V}=R^{1} p_{12 *}\left(p_{23}^{*} \mathcal{O}(\Delta) \otimes p_{3}^{*} \omega_{C}^{-1}\right) \cong p_{2}^{*} R^{1} p_{1 *}\left(\mathcal{O}(\Delta) \otimes p_{2}^{*} \omega_{C}^{-1}\right)
$$

So the Chern classes of $\mathcal{V}$ can be computed by the push forward along the first projection of the following the short exact sequence

$$
\left.0 \rightarrow p_{2}^{*} \omega_{C}^{-1} \rightarrow \mathcal{O}(\Delta) \otimes \omega_{C}^{-1} \rightarrow p_{2}^{*} \omega_{C}^{-2}\right|_{\Delta} \rightarrow 0
$$

We find

$$
0 \longrightarrow \omega_{C}^{-2} \rightarrow \mathcal{O}_{C} \otimes H^{1}\left(C, \omega_{C}^{-1}\right) \rightarrow R^{1} p_{1 *}\left(\mathcal{O}(\Delta) \otimes p_{2}^{*} \omega_{C}^{-1}\right) \longrightarrow 0
$$

Hence, $c_{1}(\mathcal{W})=4 \rho$ and $c_{i}(\mathcal{W})=0$ if $i \geq 2$.

Proof of (ii) and (iii) We want to show that

$$
\left(-4 \Theta_{1}+2 \pi-7 \rho-\zeta\right)^{5}=-5^{2} 2^{5} \zeta^{2} \rho \Theta_{1}^{2} \in H^{10}(\bar{W}) .
$$

We compute

$$
\begin{aligned}
\left(-4 \Theta_{1}+2 \pi-7 \rho-\zeta\right)^{5} & =\left(\begin{array}{l}
5 \\
3
\end{array}\right)\left(-\zeta^{3}\right)\left(-4 \Theta_{1}+2 \pi-7 \rho\right)^{2}+\left(\begin{array}{l}
5 \\
2
\end{array}\right) \zeta^{2}\left(-4 \Theta_{1}+2 \pi-7 \rho\right)^{3} \\
& =10 \cdot \zeta^{2}\left(\left(4 \rho\left(-4 \Theta_{1}+2 \pi-7 \rho\right)^{2}+\left(-4 \Theta_{1}+2 \pi-7 \rho\right)^{3}\right) .\right.
\end{aligned}
$$


The result is a combination of $\pi, \theta$ and $\rho$, which are classes of type $(1,1)+(0,2)$, $(2,0)$ and $(0,2)$, respectively. Moreover, in the proof of Lemma 4.4 we computed $\pi=\rho+\gamma$ and $\pi^{2}=\gamma^{2}=-2 \rho \Theta_{1}$. Hence, the only non-zero combinations are $\pi^{2} \Theta_{1}=-2 \rho \Theta_{1}^{2}=-2 \pi \Theta_{1}^{2}$. We find

$$
\begin{aligned}
10 & \cdot \zeta^{2}\left(\left(4 \rho\left(-4 \Theta_{1}+2 \pi-7 \rho\right)^{2}+\left(-4 \Theta_{1}+2 \pi-7 \rho\right)^{3}\right)\right. \\
& =10 \cdot \zeta^{2}\left(2^{6} \rho \Theta_{1}^{2}+3\left(-2^{4} \pi^{2} \Theta_{1}+2^{5} \pi \Theta_{1}^{2}-7 \cdot 2^{4} \rho \Theta_{1}^{2}\right)\right. \\
& =10\left(2^{6}+3\left(2^{5}+2^{5}-7 \cdot 2^{4}\right)\right) \zeta^{2} \rho \Theta_{1}^{2}=-5^{2} 2^{5} \zeta^{2} \rho \Theta_{1}^{2} .
\end{aligned}
$$

Finally, we want to show that $\int_{\bar{W}} \zeta^{2} \rho \Theta^{2}=2$. Indeed,

$$
\int_{\bar{W}} \zeta^{2} \rho \Theta^{2}=\tau_{*} \zeta^{2} \int_{\mathrm{Pic}^{1}} \Theta^{2} \int_{C} \rho=2 .
$$

This concludes the proof of the proposition.

\section{Proof of Theorem 1.2}

In this section, we prove Theorem 1.2.

Theorem The classes $\left[N_{0}\right]$ and $\left[N_{1}\right] \in H^{10}(M, \mathbb{Q})$ are linearly independent and span a totally isotropic subspace of $H^{10}(M, \mathbb{Q})$ with respect to the intersection pairing. They are given by

$$
\left[N_{0}\right]=\frac{1}{48}[F]+\beta \text { and }\left[N_{1}\right]=\frac{5}{12}[F]-4 \beta,
$$

where $[F]$ is the class of a general fiber of the Mukai system and $0 \neq \beta \in$ $\left(S^{5} H^{2}(M, \mathbb{Q})\right)^{\perp}$ satisfies $\beta^{2}=0$. As $\operatorname{deg}_{u_{1}} \beta=0$, the class $\beta$ is not effective.

From now on, all cohomology groups have $\mathbb{Q}$-coefficients.

Before coming to the proof, we want to point out, that the irreducible components over points in $\Sigma \backslash \Delta$ (see (3.1)) are of different cohomological nature. Let $D \in \Sigma \backslash \Delta$ be a reducible curve with two smooth components $C_{1}$ and $C_{2}$ meeting transversally. Then the two components $N_{1}^{\prime}$ and $N_{2}^{\prime}$ of $f^{-1}(D)$ contain an open sublocus parametrizing line bundles on $D$ of bi-degree $(2,1)$ and $(1,2)$, respectively [6, Proposition 3.7.1 and Lemma 3.3.2]. The monodromy around $\Sigma \backslash \Delta$ exchanges $C_{1}$ and $C_{2}$ and consequently the classes of the irreducible components. We find

$$
\left[N_{1}^{\prime}\right]=\left[N_{2}^{\prime}\right]=\frac{1}{2}[F] \text {. }
$$

In particular, the two components are linearly dependent. This is not true over $\Delta$.

Proposition 5.1 The classes $\left[N_{0}\right]$ and $\left[N_{1}\right] \in H^{10}(M)$ are linearly independent. 
The proof uses the following simple observation.

Lemma 5.2 Let $M \rightarrow B$ be a Lagrangian fibration and $F$ a smooth fiber. Then

$$
\left.c_{i}\left(\mathcal{T}_{M}\right)\right|_{F}=0 \text { for all } i>0 .
$$

Proof We have a short exact sequence $\left.0 \rightarrow \mathcal{T}_{F} \longrightarrow \mathcal{T}_{M}\right|_{F} \longrightarrow \mathcal{N}_{F / M} \rightarrow 0$. Now, $F \subset M$ is Lagrangian and hence $\mathcal{N}_{F / M} \cong \Omega_{F}$. Moreover, $F$ is an abelian variety and hence all its Chern classes of degree greater than zero are trivial.

Proof of Proposition 5.1 Assume that $\left[N_{0}\right]$ and $\left[N_{1}\right]$ are linearly dependent. Then there is some $\lambda \in \mathbb{Q}$ such that $[F]=\lambda\left[N_{0}\right]$, where $F \subset M$ is a smooth fiber. In particular, by the above lemma, any product of $\left[N_{0}\right]$ and the Chern classes of $M$ vanishes. However, we will show that

$$
\int_{M} c_{2}\left(\mathcal{T}_{M}\right) \cdot u_{1}^{3} \cdot\left[N_{0}\right] \neq 0
$$

leading to the desired contradiction. We have $c\left(\left.\mathcal{T}_{M}\right|_{N_{0}}\right)=c\left(\mathcal{T}_{N_{0}}\right) c\left(\Omega_{N_{0}}\right)$ and thus

$$
\left.c_{2}\left(\mathcal{T}_{M}\right)\right|_{N_{0}}=\left(2 c_{2}-c_{1}^{2}\right)\left(\mathcal{T}_{N_{0}}\right)
$$

Moreover, our computation will use the following two inputs. Let $\alpha \in H^{2}\left(\operatorname{SM}_{C}(2,1)\right)$ be the degree two Künneth component of $\left(c_{1}^{2}-c_{2}\right)\left(\mathcal{V}_{\text {univ }}\right)$ with $\mathcal{V}_{\text {univ }}$ being a universal bundle on $\operatorname{SM}_{C}(2,1) \times C$. It is known, e.g. [26, §5A], that

$$
c_{1}\left(\mathcal{T}_{\mathrm{SM}_{C}(2,1)}\right)=2 \alpha, c_{2}\left(\mathcal{T}_{\mathrm{SM}_{C}(2,1)}\right)=3 \alpha^{2} \text { and } \int_{\mathrm{SM}_{C}(2,1)} \alpha^{3}=4
$$

Further, by $[12$, Théorème $\mathrm{F}]$ it is known that $\mathcal{O}_{\mathrm{SM}_{C}(2,1)}(-2 \Theta) \cong \omega_{\mathrm{SM}_{C}(2,1)}$. Hence,

$$
\Theta=-\frac{1}{2} c_{1}\left(\omega_{\mathrm{SM}_{C}(2,1)}\right)=\frac{1}{2} c_{1}\left(\mathcal{T}_{\mathrm{SM}_{C}(2,1)}\right) .
$$

This gives,

$$
\begin{aligned}
& \int c_{2}\left(\mathcal{T}_{M}\right) u_{1}^{3}\left[N_{0}\right] \stackrel{4.10}{=} \int_{N_{0}}\left(2 c_{2}-c_{1}^{2}\right)\left(\mathcal{T}_{N_{0}}\right) \cdot(2 \Theta)^{3} \\
& =\frac{1}{2^{4}} \int_{\mathrm{SM}_{C}(2,1) \times \mathrm{Pic}^{0}} h^{*}\left(\left(2 c_{2}-c_{1}^{2}\right)\left(\mathcal{T}_{N_{0}}\right) \cdot(2 \Theta)^{3}\right) \\
& \stackrel{(4.1)}{=} \frac{2^{3}}{2^{4}} \int_{\mathrm{SM}_{C}(2,1) \times \mathrm{Pic}^{0}} p_{1}^{*}\left(2 c_{2}-c_{1}^{2}\right)\left(\mathcal{T}_{\mathrm{SM}}\right) \cdot\left(p_{1}^{*} \Theta_{\mathrm{SM}}+4 p_{2}^{*} \Theta_{0}\right)^{3} \\
& =\frac{1}{2} \int_{\mathrm{SM}_{C}(2,1) \times \mathrm{Pic}^{0}} p_{1}^{*}\left(2 c_{2}-c_{1}^{2}\right)\left(\mathcal{T}_{\mathrm{SM}}\right) \cdot\left(3 p_{1}^{*} \Theta_{\mathrm{SM}} \cdot 4^{2} p_{2}^{*} \Theta_{0}^{2}\right) \\
& =3 \cdot 2^{3} \int_{\mathrm{SM}_{C}(2,1)}\left(2 c_{2}-c_{1}^{2}\right)\left(\mathcal{T}_{\mathrm{SM}}\right) \cdot \frac{1}{2} c_{1}\left(\mathcal{T}_{\mathrm{SM}}\right) \int_{\mathrm{Pic}^{0}} \Theta_{0}^{2} \\
& =3 \cdot 2^{4} \int_{\mathrm{SM}_{C}(2,1)}\left(6 \alpha^{2}-4 \alpha^{2}\right) \alpha=3 \cdot 2^{7} \neq 0 \text {. }
\end{aligned}
$$


Proof of Theorem 1.2 We set $V:=S^{5} H^{2}(M) \subset H^{10}(M)$ so that we have an orthogonal decomposition with respect to the cup product $H^{10}(M)=V \oplus V^{\perp}$. Accordingly, we write $\left[N_{i}\right]=\alpha_{i}+\beta_{i}$ with $\alpha_{i} \in V$ and $0 \neq \beta_{i} \in V^{\perp}$ for $i=1,2$. We claim that

$$
20\left[N_{0}\right]-\left[N_{1}\right] \in V^{\perp}
$$

To see this, we decompose the second cohomology group into its transcendental and algebraic part, i.e. $H^{2}(M)=T(M) \oplus \mathrm{NS}(M)$. Now, for $i=1,2$ consider

$$
T(M) \rightarrow H^{12}(M), \quad \alpha \mapsto \alpha \cdot\left[N_{i}\right]
$$

As the symplectic form $\sigma \in T(M)$ vanishes on $N_{i}$, it follows by irreducibility of the Hodge structure $T(M)$ that the assignment (5.2) is trivial. Hence, it suffices to show that $20\left[N_{0}\right]-\left[N_{1}\right] \in\left(S^{5} \mathrm{NS}(M)\right)^{\perp}$. By (4.2) any element in $S^{5} \mathrm{NS}(M)$ is of the form $x_{1} x_{2} \ldots x_{5}$, where $x_{i}=\lambda_{M}\left(2 c_{i} . H, c_{i}, s_{i}\right)$. According to Propositions 4.10 and 4.13

$$
\int\left[N_{1}\right] x_{1} x_{2} \ldots x_{5}=-5^{2} 2^{6} \prod_{i=1}^{5} c_{i} . H=20 \int\left[N_{0}\right] x_{1} x_{2} \ldots x_{5}
$$

This proves (5.1).

Next, we write $\left[N_{1}\right]-20\left[N_{0}\right]=\alpha_{1}-20 \alpha_{0}+\beta_{1}-20 \beta_{0} \in V^{\perp}$ and conclude $\alpha_{1}=20 \alpha_{0}$. We set $\alpha=\alpha_{0}$. On the one hand, we have by Theorem 1.1

$$
2^{3}\left[N_{0}\right]+2\left[N_{1}\right]=[F]=u_{0}^{5} \in V,
$$

but also

$$
u_{0}^{5}=48 \alpha+8 \beta_{0}+2 \beta_{1}
$$

This gives $48 \alpha=u_{0}^{5}$ and $\beta_{1}=-4 \beta_{0}$. Setting $\beta=\beta_{0}$ gives the desired expression.

The last assertion follows from $\left[N_{0}\right]^{2}=\left(\frac{1}{48} u_{0}^{5}+\beta\right)^{2}=\beta^{2}$ and

$$
\left[N_{0}\right]^{2}=\int_{N_{0}} c_{5}\left(\mathcal{N}_{N_{0} / M}\right)=\int_{N_{0}} c_{5}\left(\Omega_{N_{0}}\right)=-e\left(N_{0}\right),
$$

which is known to vanish, see $[2, \S 9]$. Hence $\beta^{2}=0$, which implies $\left[N_{1}\right]^{2}=0$ and finally, as $[F]^{2}=0$, also $\left[N_{0}\right] \cdot\left[N_{1}\right]=0$.

Acknowledgements I am very grateful to Daniel Huybrechts for his invaluable support. I wish to thank Thorsten Beckmann, Norbert Hoffmann, Hsueh-Yung Lin, Georg Oberdieck, Giulia Saccà and Andrey Soldatenkov for helpful discussions and Tony Pantev for providing a secret manuscript.

Funding Open Access funding enabled and organized by Projekt DEAL. 
Open Access This article is licensed under a Creative Commons Attribution 4.0 International License, which permits use, sharing, adaptation, distribution and reproduction in any medium or format, as long as you give appropriate credit to the original author(s) and the source, provide a link to the Creative Commons licence, and indicate if changes were made. The images or other third party material in this article are included in the article's Creative Commons licence, unless indicated otherwise in a credit line to the material. If material is not included in the article's Creative Commons licence and your intended use is not permitted by statutory regulation or exceeds the permitted use, you will need to obtain permission directly from the copyright holder. To view a copy of this licence, visit http://creativecommons.org/licenses/by/4.0/.

\section{References}

1. Arbarello, E., Cornalba, M., Griffiths, P., Harris, J.: The Geometry of Algebraic Curves I. Grundlehren der mathematischen Wissenschaften 267. Springer, Berlin (1985)

2. Atiyah, M.F., Bott, R.: The Yang-Mills equations over Riemann surfaces. Philos. Trans. R. Soc. Lond. A 308, 523-615 (1982)

3. Bayer, A., Macrì, E.: Projectivity and birational geometry of Bridgeland moduli spaces. J. AMS 27, 707-752 (2014)

4. Beauville, A., Narasimhan, M.S., Ramanan, S.: Spectral curves and the generalized theta divsor. J. Reine Angew. Math. 398, 169-179 (1989)

5. Beauville, A.: Systèmes hamiltoniens complètement intégrable associés aux surfaces K3. Symphony Mathematics 32, pp. 25-31. Academic Press, New York (1991)

6. Cataldo, M.A.de., Rapagnetta, A., Saccà, G.: The Hodge numbers of O'Grady 10 via Ngô strings. (2019). arXiv:1905.03217

7. Cataldo, M.A.de., Maulik, D., Shen, J.: Hitchin firbations, abelian surfaces, and P=W. (2019). arXiv:1909.11885

8. Chen, D., Kass, J.: Moduli of generalized line bundles on a ribbon. J. Pure Appl. Algebra 220(2), 822-844 (2016)

9. Donagi, R., Ein, L., Lazarsfeld, R.: Nilpotent Cones and Sheaves on K3 Surfaces. Contemporary Mathematics 207, pp. 51-61. AMS, Providence (1997)

10. Donagi, R., Tu, L.: Theta functions for SL $(n)$ versus GL (n). Math. Res. Lett. 1, 345-357 (1994)

11. Donagi, R., Pantev, T., Simpson, C.: Geometric Langlands Higgs bundles for curves of genus 2 (in preparation)

12. Drezet, J.-M., Narasimhan, M.S.: Groupe de Picard des variétés des modules de fibrés semi-stables sur les courbes algébriques. Invent. Math. 97, 53-94 (1989)

13. Eisenbud, D.: Commutative Algebra with a View Toward Algebraic Geometry. GTM 150. Springer, Berlin (1994)

14. Hausel, T.: Global topology of the Hitchin system. Handb. Moduli II, 29-69 (2013)

15. Hitchin, N.: Critical loci for Higgs bundles. Commun. Math. Phys. 366(2), 841-864 (2019)

16. Huybrechts, D., Lehn, M.: The Geometry of Moduli Spaces of Sheaves. Cambridge Mathematical Library, 2nd edn. Cambridge University Press, Cambridge (2010)

17. Huybrechts, D.: Fourier-Mukai Transforms in Algebraic Geometry. Oxford Mathematical Monographs. The Clarendon Press, Oxford University Press, Oxford (2006)

18. Huybrechts, D.: Lectures on K3 Surfaces. Cambridge Studies in Advanced Mathematics 158. Cambridge University Press, Cambridge (2016)

19. Lehn, M.: Geometry of Hilbert schemes. CRM Proc. Lect. Notes 38, 1-30 (2004)

20. Le Potier, J.: Faisceaux semi-stables de dimension 1 sur le plan projectif. Rev. Roumaine. Pures Appl. 38(7-8), 635-678 (1993)

21. Matsushita, D.: Equidimensionality of complex Lagrangian fibrations. Math. Res. Lett. 7(4), 389-391 (2000)

22. Mukai, S.: Symplectic structure of the moduli space of sheaves on an abelian or K3 surface. Invent. Math. 77(1), 101-116 (1984)

23. Thaddeus, M.: Topology of the moduli space of stable bundles over a compact Riemann surface. Ph.D. thesis. St. John's College (1990)

24. Verbitsky, M.: Cohomology of Hyperkähler manifolds. Ph.D. dissertation, Havard University (1995). arXiv:alg-geom/9501001 
25. Yoshioka, K.: Brill-Noether problem for sheaves on K3 surfaces. In: Proceedings of the Workshop 'Algebraic Geometry and Integrable Systems related to String Theory', vol. 1232. pp. 109-124 (2001)

26. Zagier, D.: On the Cohomology of Moduli Spaces of Rank Two Vector Bundles Over Curves. The Moduli Space of Curves. Progress in Mathematics, vol. 129. Birkhäuser, Boston (1995)

27. Zagier, D.: Elementary aspects of the Verlinde formula and of the Harder-Narasimhan-Atiyah-Bott formula. Isr. Math. Conf. Proc. 9, 445-462 (1996)

Publisher's Note Springer Nature remains neutral with regard to jurisdictional claims in published maps and institutional affiliations. 